\title{
Uno y todo: del romanticismo al organicismo de Fichte
}

\section{Mariano Gaudio}

\section{(2) OpenEdition \\ Journals}

Edición electrónica

URL: http://journals.openedition.org/ref/552

DOI: $10.4000 /$ ref.552

ISSN: 2258-014X

Editor

EuroPhilosophie Editions

Referencia electrónica

Mariano Gaudio, "Uno y todo: del romanticismo al organicismo de Fichte », Revista de Estud(i)os sobre Fichte [En línea], 9 | 2014, Publicado el 28 diciembre 2014, consultado el 08 septiembre 2020. URL : http://journals.openedition.org/ref/552 ; DOI : https://doi.org/10.4000/ref.552

Este documento fue generado automáticamente el 8 septiembre 2020.

(c) EuroPhilosophie 


\title{
Uno y todo: del romanticismo al organicismo de Fichte
}

\author{
Mariano Gaudio
}

\section{1.}

1 La idea principal del organicismo natural o social refiere a la conexión mutua entre partes que conforman una totalidad y que no pueden existir por separado o con independencia de las relaciones mutuas y de las relaciones en su conjunto. En Platón o en Aristóteles, el organicismo se estructura de modo jerárquico y estático, y su fundamento reside en la naturaleza, mientras que en el pensamiento moderno la organización social parte de la igualdad fragmentaria y se erige de modo artificial, en cuanto los átomos se asocian o disocian ocasionalmente. Por tanto, ¿cómo se obtiene un entrelazamiento tal que mantenga vinculadas a las partes sin anular la libertad de cada una, y sin convertirse en una cáscara nominal y vacía? ¿Cómo y mediante cuál vínculo estable es posible la retroalimentación recíproca en un marco de igualdad? En el presente trabajo trataremos de dilucidar las características del organicismo social que traza Fichte en el Fundamento del Derecho natural (Grundlage des Naturrechts = GNR).

2 La tensión entre un enfoque -el clásico- centrado en la unicidad y otro -el moderno-en las dificultades de conciliación de los particulares, eclosiona y se sublima en la fórmula mágica del romanticismo, el "uno y todo" (en kai pan) que se evoca en la polémica sobre el spinozismo y se agudiza poética o filosóficamente en el contexto de Fichte. Al respecto, analizaremos las especulaciones de Hölderlin, Novalis y F. Schlegel. Pero aquello que los románticos resuelven sin mayores consideraciones, la identificación de la parte con el todo y la simetría entre el macro y el microcosmos, en Schelling se despliega en términos de correlación entre lo natural y lo espiritual (2). En consonancia con este filósofo, observaremos que Fichte proyecta una visión organicista no sólo sobre el saber, sino también sobre la naturaleza, el cuerpo propio y la comunidad de seres racionales (3). Con estos elementos nos centraremos en GNR, en la trabazón 
recíproca y la conformación de una voluntad común que contiene y posibilita a las particulares (4).

3 Preliminarmente, cabe preguntarse por la relación entre Fichte y los principales exponentes del romanticismo que trataremos aquí. Aunque la cercanía no sea tan intensa como la de Schelling, que también guarda diferencias con ellos, y aunque la Doctrina de la Ciencia acentúe progresivamente un lenguaje árido y técnico-específico, el idealismo de Fichte constituye un vector que irradia cierta influencia sobre las especulaciones poéticas del círculo romántico, en especial a partir de la consideración del Yo como absoluto y las infinitas posibilidades creativas contenidas en la imaginación productiva. En esta órbita, la elevación de la instancia interior-fundante a la condición de primer principio invita a la identificación con la totalidad y, por consiguiente, con Dios, la naturaleza, la vida, etc. El presunto spinozismo invertido (o de la conciencia) de Fichte habilitaría la subsunción de todo en uno, y uno en todo, en cuanto dos caras de un mismo proceso donde las partes orgánicamente intrincadas alternan su predominio en la lucha de opuestos, y donde lo interior se revierte de inmediato en exterioridad y viceversa. El romanticismo extrae esta perspectiva de Fichte, conjugando plenitud y carencia, alegría y dolor, conciliación y fragmentación; y recrea el organicismo bajo parámetros artísticos-religiosos que a su vez conllevan una mirada histórica, política o social. No obstante, el organicismo social fichteano requiere de un sustento jurídico-político.

\section{2.}

La cuestión de la organicidad constituye uno de los tópicos esenciales del romanticismo, ya sea en clave de carencia (dolor, desgarramiento, crisis, etc.), ya sea en clave de reencuentro con la unidad originaria (alegría, reconciliación, entusiasmo por transformar). La denuncia y el énfasis sobre el vínculo entre las partes y hacia la totalidad se consuman poéticamente en Hölderlin, Novalis y F. Schlegel, y filosóficamente en Schelling. Esta temática aparece previamente en la polémica sobre el spinozismo.

En efecto, poco más de una década antes de la instancia fundacional del romanticismo (esto es, hacia 1798, en la revista Athenaeum) Jacobi desata un intenso debate sobre el sentido de la filosofía de Spinoza, en principio señalando la hipotética adhesión de Lessing a este sistema, y luego ofreciendo su propia interpretación. Jacobi inicia sus Cartas sobre la doctrina de Spinoza (1785) con un poema de Goethe titulado "Lo divino", donde los seres humanos respetan a los dioses en cuanto éstos hacen en lo grande lo que los primeros harían en lo pequeño. Esta simetría se sostiene en el lema que suscribiría Lessing y con el cual se acusaba la filosofía de Spinoza: "uno y todo". Pero Jacobi condena a este sistema que se hunde en el fatalismo y en el mecanicismo, y del cual se puede salir únicamente a través del salto morale o la fe. ${ }^{1}$ Por otra parte, Lessing concebía a Dios como una totalidad orgánica -prosigue Jacobi-, pero sin poder explicar lo que no se puede explicar, el pasaje de lo infinito a lo finito. Y Spinoza, que sí puede explicar ese pasaje, también concebía a las cosas particulares mutuamente relacionadas, “...de tal manera que una de ellas sin todas las restantes, y todas las restantes sin esa una, no pueden ni existir ni ser pensadas", porque forman una "totalidad inseparable". ${ }^{2}$ Esa totalidad viviente es Dios, donde todo y cada parte deviene 
y se funde necesariamente en Uno. Consecuentemente y más allá de las diferencias respecto de Lessing y Spinoza, Jacobi exhorta a hacerse Uno con Dios. ${ }^{3}$

6 A los ojos de Hölderlin, la atracción de Jacobi hacia Spinoza se comprende por el hallazgo de la unidad suprema que implica el abandono de la explicación. Dado que "uno y todo" se anuda con la imposibilidad de deducir algo de la nada, el pasaje de lo infinito a lo finito jamás se logra explicar, ni mediante el entendimiento ni mediante la voluntad, pues la unidad trascendental e infinita resulta incompatible con el objeto de análisis. Según Hölderlin, Jacobi ataca la doctrina de Spinoza especialmente a través del fatalismo. ${ }^{4}$ Sin embargo, se podría concebir la naturaleza de otro modo y se podría captar la unidad con otra facultad, por ejemplo, con la intuición (Einsicht), o con el sueño.

7 El regreso a la naturaleza, a Grecia o a la infancia, en Hölderlin presupone un punto de partida fragmentario -el de la planta que crece sin guía, la luz que flota errante y se apega a lo que encuentra alrededor, la búsqueda de una meta imprecisa-; se trata de un sentir la carencia en todas partes. El corte vincular con la naturaleza y con los genios de otro tiempo, se reconstituye a través de los escombros y conduce a la divinidad. ${ }^{5} \mathrm{El}$ fondo-uno, el suelo, la composición originaria, requieren de un trabajo de retorno, así como la evocación de los recuerdos de la infancia, momento donde la divinidad yace en su esplendor y donde el concepto no ha dividido las cosas. Al modo de un sueño reparador de las tormentosas realidades presentes, la infancia provee un manantial vivificante que, en el curso del mundo adulto, se reviste camaleónicamente de colores hipócritas. ${ }^{6}$

8 La misma regresión hacia la unidad se complementa con la crítica a la época moderna que erradica los dioses y afirma una subjetividad que sólo se ocupa de sí misma. Los dioses duermen en lo profundo de la tierra y renacen en primavera, desde abajo y como "alma del pueblo". ${ }^{7}$ El cruce entre cielo y tierra, o la misma figura de Hiperión, se desarrolla en clave de recomposición de lo fragmentario, en una mezcla entre fuga interior y aniquilación de sí, que a su vez significa un modo de reencontrarse y reconciliarse consigo mismo. El aislamiento de Hiperión se revierte con el florecer de la plenitud vital que sacia la indigencia propia y realza el vínculo macro y microcósmico, tal como lo expresa Hölderlin en el conocido pasaje: "Ser uno con todo es la vida de la divinidad, es el cielo del hombre. Ser uno con todo lo que vive; reaparecer, en un feliz olvido de sí mismo, en el todo de la naturaleza". 8

9 La captación de la unidad se logra mediante el reconocimiento de aquello que contiene lo fragmentario, e incluso con el abandono de la facultad de conceptualizar y dividir: "el hombre es un dios cuando sueña, un mendigo cuando reflexiona". ${ }^{9}$ El sueño poético eleva hacia la captación de la unidad entre lo grande y lo pequeño, que no consiste en irse más allá, sino en volver, porque, como predica Hiperión: “Todo está en nosotros”", en la rejuvenecedora absorción de lo divino-natural. La exaltación del arte se condice con la apuesta a la religión en cuanto amor a la belleza, sin la cual el Estado sería un esqueleto vacío. ${ }^{11}$ Cuando los dioses y sus hijos (el arte y la religión) se retiran, tal como sucede en los tiempos modernos, el hombre se salva entregándose a la naturaleza, fuente de unidad y de vida. Hölderlin concluye: "En la disputa está mediada la reconciliación, y todo lo separado se vuelve a encontrar [...], todo es una única, eterna y ardiente vida". ${ }^{12}$

10 El entusiasmo romántico en la búsqueda de unidad contrasta con el desgarramiento, y éste sirve de diagnóstico sobre la época. En el mismo sentido de Hölderlin, Novalis 
recuerda el perdido enlace entre cielo y tierra, tanto en Grecia como en el Medioevo. Esta unidad se soslaya con la ciencia moderna y con el afán de cálculo, que reducen la naturaleza a número y medida. ${ }^{13} \mathrm{El}$ resultado es un universo mecánico, un molino sin arquitecto ni molinero, un engranaje que se disuelve a sí mismo. Los dioses huyeron, y el hombre quedó atrapado en la finitud, las ganancias o los honores; el hombre moderno ignora su interior e impera el negocio.

11 Tanto la Reforma como la Revolución Francesa muestran -según Novalis- la corrupción de una época cuya redención se encuentra en el espíritu de la infinitud interior, en el nuevo cristianismo basado en la libertad..$^{14} \mathrm{La}$ exaltación de la naturaleza contrasta con la crítica social al mundo burgués y su fragmentación, y lo mismo vale para la fuga interior, para el "tedio infinito" que surge del alejamiento de la bella unidad clásica y las infinitas posibilidades creativas del hombre. ${ }^{15}$ En este sentido, Novalis insiste en la identidad macro-microcósmica: "ser hombre es tanto como ser Universo". Al igual que Hölderlin, afirma: "Soñamos viajes a través del Universo, pero ¿no está el Universo dentro de nosotros? No conocemos las profundidades de nuestro espíritu...", donde reside precisamente la unidad perdida: "En ninguna parte sino dentro de nosotros está la eternidad con sus mundos". ${ }^{16}$

Los poetas, signados por la inspiración, se enamoran de sus asertos, y corresponde a la tarea filosófica la reconstrucción de sus argumentos. En el caso de F. Schlegel encontramos no sólo un tono más reposado, sino también una concepción sobre la poesía romántica, un explorar que tal vez arroje precisiones sobre la articulación unotodo. Según Schlegel, la poesía romántica aspira a unificar los distintos géneros literarios, vinculándose con la filosofía y con la retórica, fusionando creatividad y crítica; en suma, aspira a poetizar la sociedad. En este sentido, abarca todas las producciones y pretende abrazar lo vital, lo universal y lo ínfimo. Incluso la crítica se extiende al meollo del movimiento mismo, dado que algunos artistas -dice Schlegelhan quedado atrapados en el mundo interior, sin reconstruir desde allí "un espejo de todo el mundo circundante, un cuadro de la época". ${ }^{17}$

Como anticipándose a la crítica de Hegel o de Schmitt, Schlegel subraya que la fuga interior no representa sino un camino para expresar poéticamente la realidad y la época. Pero este espejo o cuadro sólo se consuma de manera progresiva, como un fluir continuo: "La poesía romántica es una poesía universal progresiva [...]. Esta poesía es la única infinita y la única libre, haciendo valer como única ley el que el arbitrio del poeta no tolera sobre sí ninguna ley". ${ }^{18}$ (La progresión y la carencia de ley jamás complacerían a Hegel o a Schmitt). La libertad absoluta para la creatividad no se direcciona unilateralmente, desde el trasfondo creativo y hacia el mundo, sino también como absorción y expresión poética de aspectos vitales como el trato, el amor o la amistad.

En Lucinda, Schlegel bucea en las turbulencias del ánimo y sus vicisitudes, en la capacidad creativa del sueño para separarse de la realidad y contemplarla de otra manera. Aquí "uno y todo" significa crear y destruir, ${ }^{19}$ éste es el cauce de la vida y de la naturaleza. Por un lado, se produce la identidad consigo mismo y con el objeto de amor o amistad, y esta unificación refleja una plenitud que disuelve "lo mío y lo tuyo"; en la fusión interior se consuma la totalidad: "Como en lo pequeño, creo yo, así es también en lo grande". ${ }^{20}$ Por otro lado, el artista romántico debe fugarse de los prejuicios y convenciones de la cultura burguesa para recuperar la inocencia infantil y recogerse en la naturaleza. Este desprecio se condice con el elogio del "puro vegetar", el ocio contemplativo, en contraste con la ambición y el afán de progreso que conducen a la 
época al aburrimiento y la fealdad. ${ }^{21}$ Este distanciarse irónico habilita la reintegración de lo fragmentario, el "uno y todo": "Nosotros veremos alguna vez en Un espíritu que somos flores de Una planta o pétalos de Una flor". ${ }^{22}$ Sin embargo, para Schlegel esta captación de la unidad se da junto con el continuo devenir de la naturaleza, es decir, las infinitas posibilidades creativas del hombre nunca alcanzan a concretizarse definitivamente en las representaciones poéticas; aunque contenemos el germen de todo, nos fragmentamos. ${ }^{23}$ De ahí que el romanticismo se proponga como ensayo, configuración o espíritu que se realiza perpetuamente a través de la letra.

En consonancia con la progresión incapaz de colmarse, en sus inicios Schelling equipara el "uno y todo" con el Yo absoluto, ${ }^{24}$ el primer principio, y describe la exaltación romántica como la expresión de una verdad "presentida y sentida", ${ }^{25}$ a saber, la contención de lo sensible, natural y terrestre, en lo suprasensible, sobrenatural y celestial. Posteriormente, en su concepción de la naturaleza Schelling desplaza las consideraciones de Fichte sobre el Yo al ámbito de su contrapolo, y argumenta que el alma, que intuye sus productos y se intuye y produce a sí misma, es una naturaleza que se organiza a sí misma. ${ }^{26}$ En este contexto presenta algunos rasgos de la organización; por ejemplo: nada puede venirle de afuera mecánicamente, se trata de un todo completo y perfecto que contiene en sí su origen y finalidad, se estructura en niveles que van de lo simple a lo complejo y que manifiestan la correlación entre naturaleza y espíritu, y en cada organización se reproduce un mundo unificado. ${ }^{27}$ Así, la planta simboliza la inteligencia, porque se apropia de la materia y la configura como si estuviera preformada para ella; la inteligencia produce la materia y la forma desde sí misma. ${ }^{28} \mathrm{En}$ este sentido, afirma Schelling: "la organización en general no es sino la imagen reducida y, por así decirlo, contraída del universo". ${ }^{29}$ Además, en la organización cada parte es lo que es en virtud del todo (y no podría serlo por separado), y el todo se explica por sí mismo. ${ }^{30}$

La conexión sistemática de la naturaleza se condice, por correlación, con el ámbito del espíritu. Si espíritu y naturaleza operan con la misma dinámica interna, la tarea de la filosofía consistirá en mostrar esa correlación, que a su vez es inherente al absoluto, al verdadero primer principio que se manifiesta en los dos ámbitos. En este sentido, para Schelling los sistemas filosóficos son aproximaciones a un modelo originario universal, variaciones que no se ensamblan entre sí en una serie infinita, sino que corresponden a la idea de organización en el que cada miembro constituye un medio y un fin, fundamento y consecuencia, un germen que contribuye en la interrelación mutua al desarrollo progresivo y paulatino de la filosofía. ${ }^{31}$

17 En suma, para los románticos el mundo sin dioses refleja la fragmentación sin unidad, ante lo cual el artista responde con la fuga interior, la reconciliación con la naturaleza y la vida, o la transformación progresiva. Se trata de un diagnóstico crítico de la modernidad y de una inmediata resolución poética. Al trasladar el en kai pan al Yo, Schelling lo dota de cierto alcance trascendental; luego, basándose en la correlación, despliega el organicismo en el plano de la naturaleza. En este magma de ideas flotaba la figura de Fichte.

\section{3.}

Así como Schelling se propone una concepción organicista no sólo de la naturaleza, sino también del espíritu y de la filosofía, del mismo modo Fichte intenta presentar su 
Doctrina de la Ciencia en términos organicistas y la extiende al ámbito de la naturaleza y de la sociedad. En efecto, en Sobre el concepto de la Doctrina de la Ciencia, una obra de notable influencia en el círculo romántico, Fichte concibe su propio sistema de la siguiente manera: "Una ciencia debe ser algo Uno, un todo" ${ }^{32}$, donde cada parte es lo que es en virtud de su posición y relación con cada una de las demás, y donde todas ellas se conectan con una proposición o principio fundamental. Este primer principio se legitima por sí mismo y transfiere su certeza como contenido y enlace con las partes conectadas. En consecuencia: "la Doctrina de la Ciencia tiene absoluta totalidad. En ella uno conduce a todo y todo a uno". ${ }^{33}$ Ahora bien, ¿se trata de una unidad mecánica, en la que cada pieza del engranaje conduce deductivamente a otra y cada una de ellas permanece independiente de sí, aunque estén trabadas por el funcionamiento?

En la Doctrina de la Ciencia nova methodo Fichte despeja las connotaciones mecanicistas y de artificialidad del saber, a la vez que amplía su concepción organicista a otros temas. Aquí explicita que la Doctrina de la Ciencia no representa un saber de conceptos muertos y estancos, sino de "algo viviente y autoactivo" ${ }^{34}$ donde fluye el significado de las palabras. La facultad que compone y permite captar los elementos en el todo es la imaginación. En el mismo respecto, el Yo no piensa e intuye por separado (como si lo espiritual y lo corporal fuesen compartimentos separados), sino como unidad o "síntesis fundamental" que engendra la autoconciencia. ${ }^{35} \mathrm{En}$ esta síntesis fundamental cada punto de la conciencia está, como mediador y como mediado, en relación con los otros puntos, y está directa o indirectamente engarzado e interrelacionado con cada otro punto y con la totalidad. De ahí que Fichte afirme que la Doctrina de la Ciencia se distingue de los restantes sistemas filosóficos: “...en que en ella no se presenta un simple pensar que está en una serie MECÁNICA [...] sino un pensar compuesto en una serie orgánica". ${ }^{36}$ Mientras que las físicas habituales conciben a la naturaleza operando mecánicamente, la visión fichteana de la naturaleza consiste en un organismo en cuyos puntos se da la concentración de las fuerzas. Lo mismo sucede en la metafísica, que reconduce a las metafísicas habituales a una síntesis fundamental, y que se reconstruye tal como las hojas, los frutos y las flores, reciben del árbol su fuerza orgánica.

Las referencias organicistas en Fichte se extienden a otros ámbitos. Quizás el mejor ejemplo de la amalgama entre naturaleza y libertad se manifiesta en el cuerpo propio, la expresión material-natural del querer. Por una parte, la organización del cuerpo refiere a la disposición natural (por ejemplo, la digestión o la circulación de la sangre); por otra, la articulación refiere a la disposición voluntaria o libre uso de los miembros. El cuerpo propio se configura como un todo organizado y articulado, a través del cual el Yo se realiza bajo ciertas condiciones y, por ende, conjuga libertad y limitación. ${ }^{37}$ Que el cuerpo sea naturalmente dado no significa que el Yo esté encorsetado en la naturaleza, sino al revés, significa que el mundo de los objetos -dice Fichte- aparece organizado gracias a la transferencia de la libertad; tampoco significa que la naturaleza sea algo extraño y ajeno, sino que está intrínsecamente vinculada con la espiritualidad. ${ }^{38}$ Sólo de esta manera el mundo adquiere sentido y vitalidad, y sólo de esta manera hay un mundo.

21 Pero la relación parte-todo depende de la perspectiva con que se lo enfoque. Así, por ejemplo, el Yo se configura individualmente en cuanto se relaciona y contrapone a otros seres racionales, sus iguales, sin los cuales no se reconocería como Yo. ${ }^{39}$ Coherentemente, Fichte sostiene que el punto "firme" y "supremo" de su indagación reside en el "reino de seres racionales", ${ }^{40}$ en la comunidad, de la que se desprende el 
individuo como parte de un todo, y de la que extrae y delimita una esfera para la realización de su libertad. ${ }^{41} \mathrm{El}$ individuo puede aparecer como todo o parte según el punto de vista. Lo mismo sucede con el cuerpo propio. Dice Fichte:

"Muevo mi brazo; éste es para sí de nuevo un todo, pero al mismo tiempo también es parte de un todo superior, de mi cuerpo propio, etc. Ciertamente también así se comporta mi cuerpo propio [en relación] con los cuerpos de los otros seres racionales exteriores a mí". ${ }^{42}$

En la comunidad se erige un cuerpo social, una articulación de seres racionales. Si el brazo no puede vivir por sí mismo sino que, como la mano en Aristóteles, obtiene su subsistencia de la totalidad, entonces el individuo tampoco podría desprenderse del cuerpo social.

Además, las connotaciones organicistas aparecen también en la naturaleza. Para Fichte la naturaleza es y se pone por sí misma, existe absolutamente y es lo que es porque es. Estos rasgos la asemejan notoriamente al Yo absoluto. Sin embargo, Fichte rechaza la división de Spinoza entre natura naturans y natura naturata, dado que considera que la naturaleza abarca producción y producto, al igual que la autoconciencia. La diferencia estriba en que la naturaleza produce ciegamente, sin libertad ni mismidad, sin ser "para-sí". Aunque el individuo nazca como parte y producto de la naturaleza (un todo organizado y sometido a la legalidad), la razón lo vivifica y determina más precisamente, lo configura como parte y producto de un Yo compartido. ${ }^{43}$ En la unidad natural las partes existen gracias al todo, y no podrían subsistir por separado: "El UNIVERSO completo también es un todo ORGANIZADO, como lo es el cuerpo propio de mi ser racional individual" ${ }^{44}$ Uno y todo; la naturaleza funciona produciéndose y haciéndose a sí misma, de modo análogo a la libertad. Pero esta unicidad sólo se fundamenta espiritualmente (no en el plano del ser, sino en el del saber).

En consecuencia, los casos examinados (el saber, el cuerpo propio, el cuerpo social, el reino racional, la naturaleza) poseen un aspecto común relevante para el organicismo político-jurídico: la dependencia recíproca de las partes converge en un punto soberano y primero, una totalidad sin la cual las partes no podrían ser lo que son y desde la cual se funda el entramado de relaciones y elementos que configuran las unidades intrínsecas.

\section{4.}

Pese a que Fichte advierte que el punto de partida de GNR, la Yoidad o racionalidad, requiere de una elevación originaria o abstracción del propio Yo, ${ }^{45}$ suele interpretarse esta obra anclada en el ser racional finito que se enfrenta al otro como obstáculo o inhibidor de su actividad. Sin embargo, si se enfoca, no la parte fragmentada, sino el todo de relaciones, la delimitación resulta fundamental para el despliegue de un trato recíproco libre y racional. En la reciprocidad descansa la relación jurídica, y desde ella se revela el concepto de comunidad. Los sujetos -dice Fichte- están vinculados y obligados por un concepto común "...en el cual dos conciencias se reúnen en una". ${ }^{46}$ En la reciprocidad se disuelve "lo mío y lo tuyo", porque se resuelve la individualidad y prima lo compartido, la ley común. Así, dos sujetos que se reconocen y tratan en condiciones de igualdad, muestran que entre ellos media una ley racional internalizada, la ley de concordancia o consecuencia ${ }^{47}$ que constituye la columna vertebral de la comunidad. 

perfecta, pero es algo más". ${ }^{.1}$ Como observamos, el cuerpo propio no se limita a la organización, sino que incluye la articulación y el movimiento libre, aspectos asimilables a los animales. A diferencia de éstos, el hombre alberga una posibilidad abierta al infinito, una determinabilidad en el proceso de formación. Su gran tesoro consiste en ser incompleto, inacabado, variable, esbozado, etc. En esta "nada" originaria reside su apertura de posibilidades y la capacidad de hacerse a sí mismo y por sí mismo, generando la autorreferencialidad o "para-sí". Desde luego, el autohacerse sería imposible fuera de la comunidad. Que la naturaleza retire su mano productora para que el hombre se forme o la cultura complete la tarea, significa no sólo que debe darse una finalidad propia, sino también que ese completarse se logra con los otros, sus iguales. ${ }^{52}$

En este punto cabe reformular la cuestión anterior, a saber, si el organicismo social se corresponde con la naturaleza o con el producto técnico. La clave se encuentra en la voluntad, que anticipamos con la explicitación de la ley común entre dos sujetos que se tratan y reconocen recíprocamente; la clave se encuentra en el querer concordante sobre el cual se sustenta toda comunidad posible. ${ }^{53}$ Pero si la coincidencia depende sólo 
de la buena fe o confianza entre las partes, lo común queda supeditado a la contingencia; así, ¿cómo se alcanzaría un enlace estable, un atenerse a la ley? Para erradicar la vulnerabilidad del trato recíproco se requiere una ley que garantice absolutamente los derechos de las partes. Esta ley tiene que ser dotada de un poder tal que su aplicación incida sobre la realidad, tiene que poder efectivizarse, e incluso erigirse más allá de las partes involucradas como "poder soberano" o "supremo". ${ }^{54}$

31 Por consiguiente, el enlace comunitario descansa en un querer jurídico fundamental. Sin embargo, a este querer fundamental no se llega -según Fichte- sumando voluntades particulares y egoístas, sino subsumiendo lo particular en una instancia universal que, en rigor, la posibilita. Del mismo modo que no se obtiene un cuerpo vivo ensamblando las partes de distintos cuerpos, jamás se obtendrá la voluntad común del agregado atómico de voluntades heterogéneas. La diferencia entre "voluntad de todos" y "voluntad general" 55 que Fichte toma de Rousseau, refiere a la prioridad de la unidad orgánica del cuerpo social. No se trata de buscar la unidad, sino de revelarla como instancia que posibilita el desacuerdo particular. En este sentido, la voluntad particular se descubre, ante todo, formando parte de la voluntad del ser-común o República, y secundariamente deviene voluntad particular-privada en la medida en que no concuerda con la ley o entabla un litigio con otro. Por tanto, la ley jurídica apunta a:

“...que las voluntades particulares se entrelacen en unidad con la voluntad de la comunidad. Esto tiene que seguirse necesariamente...", y aclara más abajo: "que la ley se presente en todos los casos y con seguridad en su aplicación, no tiene que depender en absoluto de la casualidad, o de la buena voluntad de otros, etc., sino que tiene que seguirse de la organización del todo". ${ }^{56}$

32 En el pasaje citado Fichte muestra su preocupación por que la ley sea efectiva, se aplique necesariamente, porque en ella se juega la consistencia del conjunto. Si tal efectividad no puede depender de la casualidad, de las buenas intenciones o de la limitación moral, entonces resulta necesario cristalizar el querer común en un poder capaz de ejercer, cumplir y tornar cumplible, la ley común. Con este razonamiento Fichte evita tanto la consideración meramente conceptual o formal del derecho, como la construcción del Estado a partir de condiciones preexistentes. ${ }^{57}$

33 Aunque la ley se vuelva más evidente en el incumplimiento que en el cumplimiento, éste es vital en la organización de la voluntad común. Precisamente, la diferencia entre todos (Aller), la unidad imaginada o compositum, y la totalidad (Allheit), la unidad real o totum, reside en la necesaria convergencia en un punto soberano que posibilita que cada parte esté enlazada y vinculada efectivamente con todas las demás. En el Estado se reúne lo que la naturaleza había dividido en múltiples individuos: la racionalidad. El Estado es la institución que concretiza esa unidad y que se sostiene en la vitalidad de la voluntad común. ${ }^{58} \mathrm{Al}$ respecto, Fichte apela a un ejemplo natural: el árbol. Si cada parte del árbol tuviera conciencia y voluntad, sabría que querer su autoconservación equivale a querer la conservación del todo, y que lo que afecta a una parte también afecta a las restantes. No sucede lo mismo con la arena, que mantiene su independencia. En cuanto todos son uno, el todo se convierte en totalidad.

34 En consecuencia, Fichte considera que es el primero en pensar el Estado como unidad orgánica que recupera el vínculo necesario que atraviesa a los individuos, el totum que se refleja en el producto organizado de la naturaleza, en el que “...cada parte puede ser lo que es sólo en este enlace y fuera de este enlace no lo sería en absoluto; sí, fuera de todo enlace orgánico no sería absolutamente nada". ${ }^{59}$ Gracias a la unidad en el Estado cada individuo alcanza una determinada posición, fuera de la cual su vida quedaría a 
merced de la contingencia. El hombre aislado-atómico depende únicamente de sí mismo para satisfacer sus necesidades, mientras que el ciudadano actúa o se autolimita, no directamente por un bienestar propio, sino gracias a su relación con los demás. El ciudadano se concibe y actúa vinculado y atravesado por la comunidad de pertenencia, vive contenido en la totalidad. Agrega Fichte:

"En el cuerpo orgánico, cada parte conserva continuamente al todo y está conservada ella misma en cuanto lo conserva; del mismo modo se relaciona el individuo con el Estado [...]. Cada parte, o cada ciudadano se conserva a sí mismo sólo en la posición determinada para él mediante el todo; así precisamente conserva en su parte el todo. Y ciertamente, porque el todo conserva cada parte en su posición, el todo vuelve en y sobre sí mismo, y se conserva a sí mismo". ${ }^{60}$

La totalidad social ofrece, como la naturaleza, un vínculo de contención para los particulares, no sólo limitándolos y unificando sus aportes, sino también posibilitando la condición de cada uno. Así como las partes no subsisten por separado, sino gracias a la interrelación, del mismo modo la totalidad no se produce agregando piezas, ni sumando fuerzas, sino confluyendo lo particular en lo universal. Y esta totalidad no se limita a un sueño poético, ni a un programa ético-utópico, sino que se concretiza en una institución efectiva. Y esta institución se organiza de determinada manera, nombrando funcionarios y potestades jurídicas, pero preservando siempre la igualdad de sus componentes.

\section{5.}

Una modernidad sin dioses ni cielo, signada por la fragmentación y el cálculo, no se vitaliza mediante el mero entusiasmo subjetivo o la creatividad poética, y aunque sus enlaces se construyan y destruyan -como afirma Schlegel-, es necesario ofrecer una perspectiva social con anclaje en el ámbito jurídico-político. Desde este punto de vista, la sagacidad de la crítica romántica contrasta con su propuesta de reintegración. El sueño, la fuga interior, la alternancia de opuestos, etc., no alcanzan para reconciliar lo múltiple con la unidad o para mostrar el vínculo inescindible entre partes y todo. Coherentemente, Schelling presenta el problema en el plano de la fundamentación filosófica, primero en la órbita del idealismo de Fichte y luego en el de la correlación entre lo natural y lo espiritual que lo llevará a su filosofía de la identidad. En ambos casos, el organicismo está supeditado a un primer principio, a una filosofía de lo absoluto que exponga el punto último sobre el cual reposa el entramado.

Asimismo, en Fichte la organicidad se conjuga con un primer principio del saber, y las partes se organizan en consonancia con el todo, tanto en el cuerpo propio como en el cuerpo social, en el individuo y en el reino de seres racionales, en la naturaleza y en el espíritu. La vitalidad atraviesa los elementos y fluye en sus articulaciones. En los distintos casos la libertad se armoniza con la limitación, y la parte se define desde la unidad de pertenencia. No se escinde, no se absolutiza, sino que se desprende manteniendo el vínculo, y realiza su autodeterminación desde la totalidad. Se trata, entonces, de una libertad relativa o relacional.

Lo mismo sucede en la trabazón recíproca que Fichte teje en GNR, partiendo de lo conceptual para problematizar la aplicación del derecho. En este plano resulta evidente la imposibilidad de construir un ser-común desde la sumatoria de piezas fragmentarias y dispersas, así como es insoslayable el conflicto reducido a la contienda horizontal 
entre particulares. Pero aquí las partes no replican las jerarquías naturales de los clásicos, sino que se erigen en estrictas condiciones de igualdad, porque se constituyen entre sí, porque su propia libertad se realiza con y mediante el otro, o porque la particularidad sólo se comprende como tal bajo un marco universal-inmanente. Por tanto, la unidad tiene que primar, no de modo extrínseco, sino en cuanto fuerza interna que posibilita y posiciona a cada uno de los elementos que la conforman. Tampoco ha de aparecer como una expresión de deseos, una utopía o eticidad reconciliadora, sino como una institución efectiva, concreta, capaz de sostener y garantizar el organicismo comunitario. De ahí que el Estado, aunque en una primera instancia parezca centrarse en funciones meramente coactivas y de resguardo de la seguridad, en una profundización ulterior exhibe funciones sociales de sostenimiento, de organización económica o de fomento de la cultura y la educación.

Si el organicismo social de Fichte se corresponde con el producto natural organizado, cada ciudadano es y se explica por otro ciudadano y en relación con la totalidad. Ésta no remite a un fin externo, sino a un fin interno, a la fuerza propia para producirse y conservarse, para vitalizar a sus miembros y para de ese modo vitalizarse a sí misma a través de ellos. En caso contrario, no se realizaría un Estado racional, sino despótico. Y así como el cuerpo propio preserva un espacio de libertad pero con ciertas limitaciones, del mismo modo en el cuerpo social cada miembro posee un ámbito de libertad delimitado, y en la conjugación entre libertad y limitación se asienta la justicia. La organización comunitaria se asimila notablemente a la organización natural; sin embargo, Fichte deja abierto un campo de determinabilidad para el autohacerse del hombre, una transformación que sólo se logra con el otro. Al fin y al cabo, Fichte propone cristalizar el enlace social en una institución, el Estado, sin el cual la eticidad jamás iniciaría su camino, y que sólo se podría suprimir si el hombre dejase de ser hombre.

\section{Bibliografía}

HÖLDERLIN, F., ([1796] 1914), Hyperion, oder der Eremit in Griechenland, en Sämtliche Werke und Briefe, Hrsg. F. Zinkernagel, T. II, Leipzig: Insel.

----, ([1798/1800] 1922), “Zu Jakobis Briefen über die Lehre des Spinoza”, en Sämtliche Werke. Historisch-kritische Ausgabe, Hrsg. N. Hellingrath u.a., T. III, Berlín: Propyläen-Verlag.

-----, ([1800] 1991), “Der Archipelagus", en Poemas, introd. y trad. J. M. Valverde, Barcelona: Icaria.

3 JACOBI, F. H. ([1785] 2013), Cartas sobre la doctrina de Spinoza al Sr. M. Mendelssohn, en AAVV, El ocaso de la Ilustración. La polémica del spinozismo, selección, trad. y notas M. J. Solé, Bernal: Universidad de Quilmes / Prometeo.

NOVALIS ([1795], 1837), Hymnen an die Nacht, en Novalis Schriften, Hrsg. L. Tieck y F. Schlegel, T. II, Berlín: Reimer.

------ ([1796 s.] 2006), Gérmenes o fragmentos, trad. J. Gebser, Sevilla: Renacimiento.

5 ------ ([1799, publicado en 1826] 2007), La cristiandad o Europa, en Estudios sobre Fichte $y$ otros escritos, introd., trad. y notas R. Caner-Liese, Madrid: Akal. 


\section{NOTAS}

1. Jacobi 1785: 120-122, 134-136, 139-141. Las referencias completas se consignan en bibliografía. Optamos por indicar el año de la edición original.

2. Jacobi 1785: 199.

3. Jacobi 1785: 225.

4. Hölderlin 1798: 231-235.

5. Hölderlin 1796: 18-19.

6. Hölderlin 1796: 14-15.

7. "Nuestra generación deambula en la noche y vive sin dioses -poetiza Hölderlin-, forjándose únicamente en sus propios impulsos y escuchándose sólo a sí misma en el ruidoso taller. Pero los nacidos de la divinidad viven en lo profundo de la tierra, y en la primavera que siempre florece, aunque nadie le cante a esta fuerza vivificadora, "el alma del pueblo se reúne silenciosa en la canción más libre"; Hölderlin 1800: 102-104. En la misma sintonía describe a Alemania como una sociedad burguesa donde los hombres desgarrados (que se dedican a un oficio exclusivamente, pero no son íntegros), oprimidos y sin espíritu, lo rebajan todo a la necesidad y al propio beneficio. En un pueblo sin poesía ni poetas no brota lo divino, aumenta la servidumbre, y los dioses huyen. Hölderlin 1796: 200-205. Además, cuando Hiperión se anima con Alabanda en la conquista del espíritu griego, afirma: "nadie debe reconocer nuestro futuro pueblo únicamente por sus banderas; todo se tiene que rejuvenecer, todo tiene que ser distinto desde abajo [...]; nada, ni lo más cotidiano, tampoco lo más pequeño, [será] sin espíritu y sin dioses”, 1796: 146.

8. Hölderlin 1796: 13.

9. Hölderlin 1796: 14. Más abajo, en el Libro II, 71, “uno y todo" es la Belleza. Además, el anhelo de infinitud que caracteriza al hombre es irregular, como el fuego, y si pierde la fe en todo lo grande, no hay médico ni sacerdote que pueda curar la enfermedad de la época, que consiste en entregarse a lo existente sin sentir vacío ni incomodarse, sino reflejando las cosas sin belleza, 1796: 56-58.

10. Hölderlin 1796: 22. 
11. En diálogo con Diotima, Hiperión señala los hijos de la Belleza, en primer lugar el arte, en segundo la religión, y agrega: "El sabio ama la infinitud, la omnicomprensión de la belleza por sí misma; el pueblo ama sus hijos, los dioses, que se le manifiestan en múltiples figuras. También así era en los atenienses. Y sin tal amor a la belleza, sin tal religión, todo Estado es un esqueleto vacío, sin vida ni espíritu", Hölderlin 1796: 106. En otro pasaje insiste en la crítica al Estado como poder de coacción que no logra afectar (ni conviene que lo haga) la moral y las costumbres, porque el Estado no es más que "la áspera corteza del núcleo de la vida", es un muro que rodea el jardín, pero que no sirve de nada si el suelo está seco, pues para eso se requiere que la lluvia del cielo restituya la "primavera de los pueblos", 1796: 43.

12. Hölderlin 1796: 209.

13. Novalis 1795: 10: "Los dioses huyeron con su séquito. Solitaria y sin vida quedó la naturaleza. Con cadena de hierro enlazó el áspero número y la medida estricta”.

14. Novalis 1799: $246 \mathrm{~s}$.

15. En el momento de la creación, en la Tierra infinita moraban los dioses, con felicidad y embriaguez, con la Luz engendrando vida. Pero esta edad infantil del género humano se interrumpió con el Mal, la muerte, y entonces: "La ola del gozo se despedazó / contra la roca de un tedio infinito". Novalis 1795: 10. En otros pasajes también afirma: "Nada más accesible al espíritu que lo infinito" (1796 s.: 23); "La ocupación del hombre es el ensanchamiento de su existencia hacia lo infinito" (1796 s.: 27).

16. Novalis 1796 s.: 29.

17. Schlegel 1798: 130 .

18. Schlegel 1798: 130-131.

19. Schlegel 1799: 24. "Destruir y crear, uno y todo; y así que el espíritu eterno flote eternamente sobre la eterna corriente universal del tiempo y de la vida y perciba cada ola más osada antes de que se diluya [...]. Ha llegado la hora, la esencia interior de la divinidad puede ser revelada y presentada [...]. Conságrate a ti mismo y anuncia que sólo la Naturaleza es venerable".

20. Schlegel 1799: 9-11, la cita en 11. Véase también 71 y 88. Dice Hölderlin: "No tengo nada de lo que pueda decir que sea propiamente mío", 1796: 12.

21. Schlegel 1799: 25, 33-34. Véase también 93.

22. Schlegel 1799: 12.

23. “Ambos somos uno, y sólo así se convierte el ser humano en ser humano y en él mismo por completo, aun cuando se vea y se represente poéticamente también como centro del todo y espíritu del mundo. Pero ¿por qué «representa poéticamente», ya que encontramos en nosotros el germen de todo y sin embargo seguimos siendo eternamente sólo una pieza de nosotros mismos?". Schlegel 1799: 95. El pasaje armoniza con la caracterización de la poesía romántica. "Forma, inventa, transforma y mantén el mundo y sus eternas figuras en un continuo cambio de eternas separaciones y enlaces. Oculta y ata el espíritu en la letra. La letra auténtica es todopoderosa y es la varita mágica universal. Con ella, la irresistible arbitrariedad de la gran hechicera Fantasía toca el sublime caos de la Naturaleza plena y llama a la luz a la palabra infinita, que es un espejo y fiel imagen del espíritu divino, a la que los mortales llaman Universo". 1799: 25.

24. "En el Yo ha encontrado la filosofía su en kai pan", dice Schelling en Vom Ich § 12, SSW I/1 192. Utilizamos la abreviatura SSW para las obras completas de Schelling. En § 9 nota, SSW I/1 185, y tras referir a Jacobi, Schelling relaciona el "uno y todo" directamente con Spinoza.

25. SSW I/1 $215 \mathrm{n}$.

26. SSW I/1 386.

27. SSW I/1 387: "Cada organización es un mundo reunido [...]. Es un arquetipo eterno que se expresa en cada planta; entonces, en cuanto regresamos, encontramos que la organización surge sólo de sí misma y a sí misma vuelve".

28. Schelling 1800: 291. 
29. Schelling 1800: 292. Ya en los Abhandlungen Schelling establece el correlato entre Naturaleza y Espíritu (SSW I/1 373-374), y afirma: "el mundo [objetivo] mismo consiste en esta expansión y contracción del Espíritu”, SSW I/1 396, para luego referir en nota a la expansión/contracción que Jacobi (1785: 142) pone en boca Lessing refiriéndose a Leibniz.

30. SSW I/2 40-41.

31. SSW I/1 457-458.

32. UBWL $§ 1$, GA I/2 114. Citamos la obra de Fichte con las abreviaturas propias de esta revista.

33. UBWL $\S 4$, GA I/2, 131 nota. Del tema del organicismo en UBWL nos ocupamos en "La unidad sistemática y los principios de la Doctrina de la Ciencia de Fichte", en prensa.

34. WLnm-H § 16 II, GA IV/2 207.

35. WLnm-H § 16 II, GA IV/2 211-213 s., 224-225.

36. WLnm-H § 16 II, GA IV/2 226.

37. WLnm-H § 10, GA IV/2 111.

38. WLnm-H § 13, GA IV/2 155-156.

39. WLnm-H § 12, GA IV/2 141. "Lo primero y supremo que encuentro soy yo; pero no me puedo encontrar sin encontrar también otros seres racionales. 'Yo soy INDIVIDUo' no tiene sentido si no se piensa también a otro [individuo]", 143.

40. WLnm-H § 12, GA IV/2 145.

41. WLnm-H § 15, GA IV/2 177.

42. WLnm-H § 18, GA IV/2 257.

43. WLnm-H § 18, GA IV/2 258.

44. WLnm-H § 18, GA IV/2 259.

45. GNR Ein. I, GA I/3, 313.

46. GNR § 4 Cor. [I], GA I/3, 354.

47. GNR $§ 4$ Cor. [I], GA I/3, 354; Cor. [II], 358.

48. GNR Ein. II, GA I/3, 319; § 3 III-IV, 342-344; § 3 Cor., 347-348; § 4, 351.

49. GNR § 6 VII, GA I/3, 375 .

50. GNR § 6 VII e, GA I/3, 378.

51. GNR § 6 VII f, GA I/3, 379 .

52. GNR $§ 6$ Cor., GA I/3, 381 (167-168): "Cualquier animal se mueve a las pocas horas de su nacimiento. [...El hombre] necesita la libre ayuda de los hombres, y sin ella moriría poco después de nacer. Apenas ha abandonado el cuerpo de la madre, la naturaleza retira su mano de él y, por así decirlo, lo arroja afuera".

53. GNR § 7 IV, GA I/3, 387.

54. GNR \& $8 \mathrm{~V}, \mathrm{GA} \mathrm{I} / 3,399$ : Übermacht, Obergewalt. La imposibilidad de resolver el conflicto jurídico sin un poder soberano atraviesa el desarrollo del derecho originario y del derecho de coacción: § 12 IV-VI, GA I/3, 415-417; § 13, 424-425.

55. GNR § $8 \mathrm{~V}, \mathrm{GA} \mathrm{I} / 3,400$ nota.

56. GNR § $8 \mathrm{~V}, \mathrm{GA} I / 3,401-402$.

57. Para Fichte no hay relación jurídica alguna fuera del Estado: GNR § 8 III, GA I/3, 395; § 15, 432. La crítica a la filosofía jurídico-formal: Ein. II, 319 s. De este tema y de otros relacionados nos hemos ocupado en un artículo de 2010, en esta misma revista.

58. GNR § 17 IV, GA I/4, 13-14. En este pasaje Fichte sitúa al Estado en una posición relativa: por un lado, unifica a los particulares en una totalidad; por otro, esta totalidad forma parte de la razón-una de la humanidad y, por ende, la eticidad completaría la tarea de unificación y conduciría a la supresión del Estado, tal como suelen subrayar incansablemente los intérpretes de Fichte. Este aspecto amerita una discusión que excede los límites del presente trabajo, es decir, una profundización sobre el significado de la supresión; de todos modos, poco más abajo ( $\$ 17$ Cor. GA I/4, 18) abandona esta perspectiva.

59. GNR § 17 Cor., GA I/4, 19. 
60. GNR § 17 Cor., GA I/4, 19-20. Véase también: EVBG GA I/3 49, DS 102-103. GHS FSW III 399, ECC 16.

\section{RESÚMENES}

Organicism shows the connection between the parts and in relation to a whole. In this article I propose an organicist reading of Fichte's Grundlage des Naturrechts (GNR). I will firstly analyse the romantic vision of "one and all", i.e., the way of articulating unity and fragmentation according to Hölderlin, Novalis and F. Schlegel (1). After having considered the romantic artistic and religious solution to the modern crisis, I will explore the case of Schelling's identification of unity and Self and his idea of organization of the elements according to natural and spiritual correlation (2). I will then argue that Schelling's philosophical solution leads to Fichte. Fichte's organicism refers not only to knowledge, but it also encompasses body, nature and community. Fichte distinctly states that a part can only exist in relation to other parts and to the whole, and that all is to be defined from a first (sovereign) principle (3). I will show that the organicism in the GNR allows to conceive the subjects in a reciprocal relationship, the particular willing as contained in one general will, and the State as an organized natural product (point 4).

\section{ÍNDICE}

Keywords: romanticism, organicism, general will

\section{AUTOR}

MARIANO GAUDIO

Universidad de Buenos Aires 